\title{
Gravity with Extra Dimensions and Dark Matter Interpretation: A Straightforward Approach
}

\author{
C. H. Coimbra Araújo ${ }^{1}$ and Roldão da Rocha ${ }^{2}$ \\ ${ }^{1}$ Universidade Federal do Paraná (UFPR), Campus Palotina, 85950-000 Palotina, PR, Brazil \\ ${ }^{2}$ Centro de Matemática, Computação e Cognição, Universidade Federal do ABC, 09210-170 Santo André, SP, Brazil \\ Correspondence should be addressed to Roldão da Rocha; roldao.rocha@ufabc.edu.br
}

Received 8 May 2013; Accepted 6 June 2013

Academic Editors: G. A. Alves, G. Bonvicini, C. A. D. S. Pires, and A. Koshelev

Copyright (c) 2013 C. H. Coimbra Araújo and R. da Rocha. This is an open access article distributed under the Creative Commons Attribution License, which permits unrestricted use, distribution, and reproduction in any medium, provided the original work is properly cited.

\begin{abstract}
Any connection between dark matter and extra dimensions can be cognizably evinced from the associated effective energymomentum tensor. In order to investigate and test such relationship, a higher dimensional spacetime endowed with a factorizable general metric is regarded to derive a general expression for the stress tensor-from the Einstein-Hilbert action-and to elicit the effective gravitational potential. A particular construction for the case of six dimensions is provided, and it is forthwith revealed that the missing mass phenomenon may be explained, irrespective of the dark matter existence. Moreover, the existence of extra dimensions in the universe accrues the possibility of a straightforward mechanism for such explanation. A configuration whose density profile coincides with the Newtonian potential for spiral galaxies is constructed, from a 4-dimensional isotropic metric plus extradimensional components. A Miyamoto-Nagai ansatz is used to solve Einstein equations. The stable rotation curves associated with such system are computed, in full compliance with the observational data, without fitting techniques. The density profiles are reconstructed and compared to the ones obtained from the Newtonian potential.
\end{abstract}

\section{Introduction}

General relativity (GR) provides solutions to the Einstein equations that currently offer important support to understand, for instance, black holes and cosmology. Mainly in the last two decades, such formalism was extended for extradimensional spacetimes. There are many motivations to consider such approach: from the Kaluza-Klein-and string theory as well-idea of unified fields to the recent effort to deal with the hierarchy problem from the idea of diluting gravity in submillimetric extra dimensions $[1,2]$. Another motivation-indeed more fundamental for GRis that higher dimensions may play a fundamental role to unravel profound interpretations concerning the nature of spacetime. On this behalf, a series of works deal with a $(4+n)$-dimensional metric splitting, in order to find solutions for the Einstein equations, in both static or stationary spherical/axisymmetric geometries. The static, spherically symmetric solutions of Kaluza-Klein theory were calculated independently by several authors [3]. For instance, in $[4,5]$ some stationary spherically symmetric solutions on KaluzaKlein theory are provided, as well as on 5-dimensional GR [6]. Furthermore, in [7] some asymptotically flat KaluzaKlein solutions, corresponding to regular black holes in four dimensions, are regarded, and prominent features concerning thermal evaporation are presented. Such formalism is generalized in a $(4+n)$-dimensional scenario [8]. It is accomplished in the so-called abelian Kaluza-Klein theorypossessing the $\mathrm{U}(1)^{n}$ as its internal isometry group-whose lower dimensional case was investigated in $[9,10]$. The rotating dyonic black holes of Kaluza-Klein theory were investigated [11] withal, and the explicit connection between such models and extradimensional theories is explored in [12-14].

Therewith, one of the most outstanding noncompact extra dimensions usages is the Randall-Sundrum (RSI) model, wherein the so-called braneworld is embedded in an $\mathrm{AdS}_{5}$ space $[1,2]$. Moreover, some large extra dimensions 
formalisms were investigated in a $(4+1+1)$-dimensional setting. The Einstein equations with negative bulk cosmological constant and a 3-brane, with appropriately tuned energymomentum tensor, were solved [15-17]. In the case of two extra dimensions, which will be considered as a particular case of our formalism, some aspects were approached in [18].

The pivotal physical motivation in our formulation is that gravity with extra dimensions can possibly shed new light on the so-called "dark matter" puzzle [19-24]. The idea is not a novelty since some theories, as universal extra dimensions [25], and some variants of Kaluza-Klein theories as well present particles corresponding to extradimensional excitation modes, representing a dark matter candidate (for a review see [26]). Here the approach is different, in the sense that it is argued whether the existence of higher dimensional spacetimes suffices to explain the missing mass problem ( $a k a$ "dark matter"), without any specific dark particle.

In order to precisely ascertain what this assumption infuses in the dark matter problem, we delve into a generalization where our universe has $D=4+n$ dimensions. Moreover, at a first glance nothing precludes the matter to live-or leak as well-in the $n$ extra dimensions (see, e.g., at the level of particle physics, the UED proposition [25]). In what follows the formalism is developed a la Kaluza-Klein.

Accordingly from such scenario, an Einstein-Hilbert gravitational action

$$
S=\int \mathrm{d}^{4} x \mathrm{~d}^{n} y \sqrt{-^{(4+n)} g}\left({ }^{(4+n)} R+\mathscr{L}_{M}\right),
$$

where $\mathscr{L}_{M}$ denotes the matter Lagrangian, leads to the field equations

$$
{ }^{(4+n)} G_{A B}={ }^{(4+n)} T_{A B},
$$

where $A, B=0,1, \ldots, 4+n$ and $y$ denotes the extra dimensions. Hereon the tensor components ${ }^{(4+n)} G_{A B}$ and ${ }^{(4+n)} T_{A B}$ are, respectively, denoted by $G_{A B}$ and $T_{A B}$ (the same for the curvature tensor and scalar and for the metric components as well). No modification is considered for the gravitational constant $G$.

Given such approach, in Section 2 the spacetime metric is split, in order to express the 4-dimensional stress tensor. It infuses some interpretations about the extra terms, appearing due to the inclusion of new dimensions (Section 3). In Section 4 the equations of motion are developed, and in Section 5 we calculate-by linearized gravity - the Newtonian limit and a modified Poisson equation for the theory. Section 6 is dedicated to show how one can interpret dark matter as a prominent pure effect, coming from the spacetime extra dimensions. We provide particular cases where two extra dimensions are regarded. Stable galaxy rotation curves are presented together with their associated analytical expression when the system is axisymmetric in Section 7. Finally in Section 8 some results with real galaxies are presented and analyzed which, together with the results in Section 7, put our formalism in compliance with observational data.

A companion paper shows a phenomenological example [20], as well as other semiphenomenological ones provided by [19]. The assumptions here are straightforward, and quantum field theory is not explicitly taken into account. GR is the theoretical limit concerning the present work. In this paper, unless explicitly mentioned, $c=1=8 \pi G$, and the signature adopted for the 4-dimensional part of the metric is $\operatorname{diag}(-,+,+,+)$. In addition, it is considered $a b$ initio that the extradimensional part is constituted by spacelike coordinates.

\section{Splitting the Metric}

Our universe is supposed to have $3+1+n$ dimensions. Initially, the extra dimensions compactification is kept apart. The most general metric for such universe is given by

$$
g_{A B}=\left(\begin{array}{ll}
g_{\alpha \beta} & g_{\alpha b} \\
g_{a \beta} & g_{a b}
\end{array}\right),
$$

where $\alpha, \beta=0, \ldots, 3$ and $a, b=4, \ldots, n$. Furthermore we consider the convention to make the metric as a function of $3+1$ coordinates only: $g_{A B}=g_{A B}\left(x^{\alpha}\right)$. These metric components $g_{A B}$ contain the $3+1$ universe metric terms $g_{\alpha \beta}$ and the extra dimensional terms $g_{a b}$, as well as the crossed components. Equation (3) can be rewritten for convenience as

$$
g_{A B}=g_{\alpha \beta} \delta_{A}^{\alpha} \delta_{B}^{\beta}+g_{a b} \delta_{A}^{a} \delta_{B}^{b}+g_{\alpha b} \delta_{A}^{\alpha} \delta_{B}^{b}+g_{a \beta} \delta_{A}^{a} \delta_{B}^{\beta},
$$

where $\delta_{j}^{i}$ are the Kronecker symbols. The derivatives for such metric components are given by

$$
\begin{aligned}
g_{A B, C}= & g_{\alpha \beta, \gamma} \delta_{A}^{\alpha} \delta_{B}^{\beta} \delta_{C}^{\gamma}+g_{a b, \gamma} \delta_{A}^{a} \delta_{B}^{b} \delta_{C}^{\gamma} \\
& +g_{\alpha b, \gamma} \delta_{A}^{\alpha} \delta_{B}^{b} \delta_{C}^{\gamma}+g_{a \beta, \gamma} \delta_{A}^{a} \delta_{B}^{\beta} \delta_{C}^{\gamma}
\end{aligned}
$$

The case $g_{A B}=g_{\alpha \beta} \delta_{A}^{\alpha} \delta_{B}^{\beta}+g_{a b} \delta_{A}^{a} \delta_{B}^{b}$ is considered here, motivated by formalisms, where $g_{\alpha}^{a}=0$, namely, RandallSundrum warped metrics $[1,2]$ and other physically relevant cases [12-14, 21-23, 27-40]. In particular, a comprehensive program on observation and measurements on braneworld effects in astrophysics and particle physics is provided in [35, 36]. Furthermore, some generalizations regarding variable tension braneworld models and cognizable physical effects were presented [37-40].

The inverse metric is written as

$$
g^{A B}=g^{\alpha \beta} \delta_{\alpha}^{A} \delta_{\beta}^{B}+g^{a b} \delta_{a}^{A} \delta_{b}^{B},
$$

and the derivatives are straightforwardly provided by (5):

$$
g_{A B, C}=g_{\alpha \beta, \gamma} \delta_{A}^{\alpha} \delta_{B}^{\beta} \delta_{C}^{\gamma}+g_{a b, \gamma} \delta_{A}^{a} \delta_{B}^{b} \delta_{C}^{\gamma} .
$$

Assuming that the spacetime has a connection presenting no torsion, one yields the following Christoffel symbols: $\left\{\begin{array}{c}A \\ B C\end{array}\right\}=$ $(1 / 2) g^{A M}\left(g_{B M, C}+g_{C M, B}-g_{B C, M}\right)$. Splitting this last expression by (6) and (7) it reads

$$
\begin{aligned}
\left\{\begin{array}{c}
A \\
B C
\end{array}\right\}= & \left\{\begin{array}{c}
\alpha \\
\beta \gamma
\end{array}\right\} \delta_{\alpha}^{A} \delta_{B}^{\beta} \delta_{C}^{\gamma} \\
& +\frac{1}{2}\left[g^{a m}\left(g_{b m, \gamma} \delta_{a}^{A} \delta_{B}^{b} \delta_{C}^{\gamma}+g_{c m, \beta} \delta_{a}^{A} \delta_{B}^{\beta} \delta_{C}^{c}\right)\right. \\
& \left.-g^{\alpha \mu} g_{b c, \mu} \delta_{\alpha}^{A} \delta_{B}^{b} \delta_{C}^{c}\right] .
\end{aligned}
$$


The Ricci tensor components are well known to be written as

$$
\begin{aligned}
R_{A B}= & \partial_{M}\left\{\begin{array}{c}
M \\
A B
\end{array}\right\}-\partial_{B}\left\{\begin{array}{c}
M \\
A M
\end{array}\right\} \\
& +\left\{\begin{array}{c}
N \\
A B
\end{array}\right\}\left\{\begin{array}{c}
M \\
N M
\end{array}\right\}-\left\{\begin{array}{c}
N \\
A M
\end{array}\right\}\left\{\begin{array}{c}
M \\
N B
\end{array}\right\} .
\end{aligned}
$$

Taking into account that the terms of the metric depend solely on $x^{\alpha}$, the equation above reads

$$
R_{A B}=R_{\alpha \beta} \delta_{A}^{\alpha} \delta_{B}^{\beta}+R_{a b} \delta_{A}^{a} \delta_{B}^{b}
$$

The splitting (6) and the derivatives (7) of the metric in the Christoffel symbols (8) lead to the following expressions for (10):

$$
\begin{aligned}
& R_{\alpha \beta}=\mathscr{R}_{\alpha \beta}-\frac{1}{2}\left[g_{, \beta}^{m c} g_{m c, \alpha}+g^{m c} g_{m c, \alpha \beta}\right. \\
&\left.+\frac{1}{2} g^{n c} g_{m c, \alpha} g^{m e} g_{n e, \beta}\right], \\
& R_{a b}=-\frac{1}{2}\left[g_{, \mu}^{\mu \gamma} g_{a b, \gamma}+g^{\mu \gamma} g_{a b, \gamma \mu}\right. \\
&-\frac{1}{2}\left(g^{n c} g_{a c, \mu} g^{\mu \gamma} g_{n b, \gamma} g^{\nu \gamma} g_{a m, \gamma}\right. \\
&\left.\left.\quad \times g^{m c} g_{b c, \nu}-g^{\nu \gamma} g_{a b, \nu} g^{m c} g_{m c, \gamma}\right)\right],
\end{aligned}
$$

where $\mathscr{R}_{\alpha \beta}=\partial_{\mu}\left\{\begin{array}{c}\mu \\ \alpha \beta\end{array}\right\}-\partial_{\beta}\left\{\begin{array}{c}\mu \\ \alpha \mu\end{array}\right\}+\left\{\begin{array}{c}\nu \\ \alpha \beta\end{array}\right\}\left\{\begin{array}{c}\mu \\ \nu \mu\end{array}\right\}-\left\{\begin{array}{c}\nu \\ \alpha \nu\end{array}\right\}\left\{\begin{array}{c}\mu \\ \nu \beta\end{array}\right\}$ is the conventional $(3+1)$ Ricci tensor.

\section{The Energy-Momentum Tensor}

Consider now the Einstein tensor components $G_{A B}=R_{A B}$ $(1 / 2) R g_{A B}$, where $R=g^{M N} R_{M N}$ is the scalar curvature. For a stress tensor written as $T_{A B}=T_{\alpha \beta} \delta_{A}^{\alpha} \delta_{B}^{\beta}+T_{a b} \delta_{A}^{a} \delta_{B}^{b}$, one can follow the same arguments accomplished for $R_{A B}$. Introducing the results obtained in the previous Section in (2), the split components of the stress tensor are given by

$$
\begin{aligned}
& T_{\alpha \beta}=R_{\alpha \beta}-\frac{1}{2}\left(g^{M N} R_{M N}\right) g_{\alpha \beta}, \\
& T_{a b}=R_{a b}-\frac{1}{2}\left(g^{M N} R_{M N}\right) g_{a b} .
\end{aligned}
$$

The tensor $T_{\alpha \beta}$ represents the energy/pressure content in the $(3+1)$-dimensional landscape. This is the part that is clearly of paramount interest. Regardless of $T_{a b} \neq 0$, here it is assumed that only the $T_{\alpha \beta}$ visible part can bring some light to observational issues. From the expression developed for $R_{\alpha \beta}$, (11), the influence of extra dimensions is evident. From (11), (12), and (13) it yields

$$
T_{\alpha \beta}=\mathscr{T}_{\alpha \beta}+\mathfrak{I}_{\alpha \beta},
$$

where $\mathscr{T}_{\alpha \beta}$ represents the $(3+1)$-dimensional influence and $\mathfrak{I}_{\alpha \beta}$ denotes the correction elicited uniquely from extra dimensions where explicitly

$$
\begin{gathered}
\mathscr{T}_{\alpha \beta}=\mathscr{R}_{\alpha \beta}-\frac{1}{2}\left(g^{\mu \nu} \mathscr{R}_{\mu \nu}\right) g_{\alpha \beta}, \\
\mathfrak{I}_{\alpha \beta}=-\frac{1}{2}\left\{\mathfrak{R}_{\alpha \beta}-\frac{1}{2}\left(g^{\mu \nu} \mathfrak{R}_{\mu \nu}\right) g_{\alpha \beta}+\left(g^{m n} R_{m n}\right) g_{\alpha \beta}\right\} .
\end{gathered}
$$

Note that the stress $\mathscr{T}_{\alpha \beta}$ has the conventional form for the Einstein tensor $\mathscr{G}_{\alpha \beta}$. The tensor $\mathfrak{R}_{\alpha \beta}$ represents a "curvature" term evinced from the presence of extra dimensions, and is given by

$$
\mathfrak{R}_{\alpha \beta}=g_{, \beta}^{m c} g_{m c, \alpha}+g^{m c} g_{m c, \alpha \beta}+\frac{1}{2} g^{n c} g_{m c, \alpha} g^{m e} g_{n e, \beta} \text {. }
$$

In addition, from the action (1) the stress tensor can be derived from the conventional definition $T_{A B}:=-2\left(\delta \mathscr{L}_{M}\right)$ $\left.\delta g^{A B}\right)+g_{A B} \mathscr{L}_{M}$. In this new approach, it is clear that the $(3+1)$-dimensional part $T_{\alpha \beta}$ is proportional to the Lagrangian multiplied by $g_{\alpha \beta}$ and some quantity from the variation $\delta / \delta g^{A B}$.

\section{An Expression for the Equations of Motion}

A general Lagrangian for the gravitating test particles in a spacetime with extra dimensions, with metric elements $g_{A B}=$ $g_{\alpha \beta} \delta_{A}^{\alpha} \delta_{B}^{\beta}+g_{a b} \delta_{A}^{a} \delta_{B}^{b}$, can be written as

$$
L=\left(g_{A B} \dot{x}^{A} \dot{x}^{B}\right)^{1 / 2}=\left(g_{\alpha \beta} \dot{x}^{\alpha} \dot{x}^{\beta}+g_{a b} \dot{x}^{a} \dot{x}^{b}\right)^{1 / 2},
$$

where $\dot{x}^{A}=\mathrm{d} x^{A} / \mathrm{d} s$. The motion equations come from the Euler-Lagrange expression $\mathrm{d} / \mathrm{d} s\left(\partial L / \partial \dot{x}^{C}\right)-\partial L / \partial x^{C}=0$. As $\partial_{A}=\partial_{\alpha} \delta_{A}^{\alpha}+\partial_{a} \delta_{A}^{a}$ and $g_{a b}=g_{a b}\left(x^{\alpha}\right)$, it follows that

$$
\begin{aligned}
\frac{\partial L}{\partial x^{C}} & =\frac{\partial L}{\partial x^{\gamma}} \delta_{C}^{\gamma}+\frac{\partial L}{\partial x^{c}} \delta_{C}^{c}, \\
\frac{\partial L}{\partial x^{\gamma}} & =\frac{1}{2} L^{-1}\left(g_{\alpha \beta, \gamma} \dot{x}^{\alpha} \dot{x}^{\beta}+g_{a b, \gamma} \dot{x}^{a} \dot{x}^{b}\right),
\end{aligned}
$$

and $\partial L / \partial x^{c}=0$. It immediately yields

$$
\frac{\partial L}{\partial x^{C}}=\frac{1}{2} L^{-1}\left(g_{\alpha \beta, \gamma} \dot{x}^{\alpha} \dot{x}^{\beta}+g_{a b, \gamma} \dot{x}^{a} \dot{x}^{b}\right) .
$$

Likewise, the term $\mathrm{d} / \mathrm{d} s\left(\partial L / \partial \dot{x}^{C}\right)$ can be developed:

$$
\begin{aligned}
\frac{\partial L}{\partial \dot{x}^{\gamma}} & =\frac{1}{2} L^{-1}\left(g_{\gamma \beta} \dot{x}^{\beta}+g_{\alpha \gamma} \dot{x}^{\alpha}\right)=L^{-1} g_{\mu \gamma} \dot{x}^{\mu}, \\
\frac{\partial L}{\partial \dot{x}^{c}} & =\frac{1}{2} L^{-1}\left(g_{c b} \dot{x}^{b}+g_{a c} \dot{x}^{a}\right)=L^{-1} g_{m c} \dot{x}^{m} .
\end{aligned}
$$

Now

$$
\frac{\mathrm{d}}{\mathrm{d} s}\left(\frac{\partial L}{\partial \dot{x}^{\gamma}}\right)=L^{-1}\left[\left(\frac{\partial g_{\mu \gamma}}{\partial x^{\sigma}}\right) \dot{x}^{\sigma} \dot{x}^{\mu}+g_{\mu \gamma} \ddot{x}^{\mu}\right] .
$$


Since $x^{a}$ are cyclic variables, it reads the integration constant

$$
g_{c m} \dot{x}^{m}=N_{c}
$$

a constant vector. Hence $\mathrm{d} / \mathrm{d} s\left(\partial L / \partial \dot{x}^{c}\right)=0$. Inserting the terms together, multiplying by $L g^{\mu \gamma}$, and using (22), the equations of motion are derived:

$$
\ddot{x}^{\mu}+\left\{\begin{array}{c}
\mu \\
\alpha \beta
\end{array}\right\} \dot{x}^{\alpha} \dot{x}^{\beta}=\frac{1}{2} g_{a b, \gamma} g^{\mu \gamma} N_{c} g^{a c} N_{d} g^{b d} .
$$

Clearly it is possible to realize that the extra dimensions induce an external force in the system that depends on $g_{a b}$ and $N_{c}$.

\section{Equation for the Visible Field}

The main aim now is to compute the gravitational potential in the Newtonian limit since galaxies and clusters can be described physically as Newtonian objects, corresponding to the approximation in which gravity is weak. The weak limit is assumed uniquely in the 4-dimensional spacetime: the deviation $\gamma_{\alpha \beta}$ of the 4-dimensional metric $g_{\alpha \beta}=\eta_{\alpha \beta}+\gamma_{\alpha \beta}$ is small ( $\eta_{\alpha \beta}$ denotes the Minkowski metric). For the linearized gravity, the stress (15) is essentially written from linearized curvature $\mathscr{R}_{\alpha \beta}^{(1)}=\partial_{\mu}\left\{\begin{array}{c}\mu \\ \alpha \beta\end{array}\right\}-\partial_{\alpha}\left\{\begin{array}{c}\mu \\ \mu \beta\end{array}\right\}$ as

$$
\mathscr{T}_{\alpha \beta}=-\frac{1}{2} \partial^{\mu} \partial_{\mu} \bar{\gamma}_{\alpha \beta}+\partial^{\mu} \partial_{(\beta} \bar{\gamma}_{\alpha) \mu}-\frac{1}{2} \eta_{\alpha \beta} \partial^{\mu} \partial^{\nu} \bar{\gamma}_{\mu \nu}
$$

where $\bar{\gamma}_{\alpha \beta}=\gamma_{\alpha \beta}-(1 / 2) \eta_{\alpha \beta} \gamma$ is the traceless part of $\gamma_{\alpha \beta}$ and $\gamma=\gamma_{\alpha}^{\alpha}$. Linearized gravity has a gauge freedom given by $\gamma_{\alpha \beta} \mapsto \gamma_{\alpha \beta}+\mathfrak{E}_{\xi} \eta_{\alpha \beta}$, where $\mathfrak{E}_{\xi}$ denotes the Lie derivative with respect to the generators $\xi^{\alpha}$ of a differential diffeomorphism. To the first order, such transformation represents the same physical transformation as $\gamma_{\alpha \beta}$. This gauge freedom is used to simplify the linearized Einstein equation. Solving the equation $\partial^{\beta} \partial_{\beta} \xi_{\alpha}=-\partial^{\beta} \bar{\gamma}_{\alpha \beta}$ for $\xi_{\alpha}$, a gauge transformation [41] that leads to $\partial^{\beta} \bar{\gamma}_{\alpha \beta}=0$-similar to the Lorentz gauge condition-can be elicited to obtain the simplified Einstein equation

$$
\mathscr{T}_{\alpha \beta}=-\frac{1}{4} \partial^{\mu} \partial_{\mu} \bar{\gamma}_{\alpha \beta}
$$

For the extra part it reads

$$
\mathfrak{I}_{\alpha \beta}=\frac{1}{2}\left[\frac{1}{2}\left(g^{m n} \partial^{\mu} \partial_{\mu} g_{m n}\right) g_{\alpha \beta}-g^{m n} g_{m n, \alpha \beta}\right] .
$$

When gravity is weak, the linear approximation to GR should be valid. There exists a global inertial coordinate system of $\eta_{\alpha \beta}$ such that

$$
\begin{gathered}
T_{\alpha \beta}=\mathscr{T}_{\alpha \beta}+\mathfrak{T}_{\alpha \beta} \approx \rho t_{\alpha} t_{\beta} \\
-\frac{1}{4} \partial^{\mu} \partial_{\mu} \bar{\gamma}_{\alpha \beta}+\frac{1}{2}\left[\frac{1}{2}\left(g^{m n} \partial^{\mu} \partial_{\mu} g_{m n}\right) \eta_{\alpha \beta}-g^{m n} g_{m n, \alpha \beta}\right] \\
=\rho t_{\alpha} t_{\beta},
\end{gathered}
$$

where $t_{\alpha}$ is the time direction associated with this coordinate system. This equation can be interpreted as the modified Poisson equation considering a universe with more than $3+1$ dimensions.

\section{Extra Dimensions and the Interpretation of Dark Matter}

Define $\bar{\gamma}_{\alpha \beta} \equiv-4 \phi$, where $\phi=\phi(\vec{x})$ is a 3 -space scalar field. Furthermore consider a line element $\mathrm{d} s_{n}^{2}=\sum_{i=1}^{n} e^{\psi_{i}} \mathrm{~d} z_{i}^{2}$, where $\mathrm{d} s_{n}^{2}$ is the world line for the extra sector, $z_{i}$ denotes the extra coordinates, and $\psi_{i}=\psi_{i}(\vec{x})$ are potentials associated to extra dimensions.

If one asserts, as a first approximation, that $R_{a b}=0$ (i.e., not similar to impose vacuum in the extra space, since now $T_{a b}$ becomes $\left.T_{a b}=-1 / 2\left(g^{M N} R_{M N}\right) g_{a b}\right)$, (12) implies at first order the sigma model $g^{\mu \nu}\left(\sigma_{, \mu} \sigma^{-1}\right)_{, v}=0$ for the extra part, where $\sigma$ denotes the diagonal matrix representing the metric associated with the system, which implies

$$
\partial^{\mu} \partial_{\mu} g_{a b}=0
$$

yielding the following equation:

$$
-\frac{1}{4} \partial^{\mu} \partial_{\mu} \bar{\gamma}_{\alpha \beta}-\frac{1}{2} g^{m n} g_{m n, \alpha \beta}=\rho t_{\alpha} t_{\beta}
$$

or in other words

$$
\nabla^{2} \phi=\rho
$$

It means that our visible matter density profile is provided uniquely by the 4-dimensional field. If extra dimensions have some impact on the theory, certainly it is not as extra matter, but it appears at least as the term $(1 / 2) g^{m n} g_{m n, \alpha \beta}$ in (29). In matter models applicable to a galactic context pressure arises from the velocity dispersion in the motion of particles or from exchange of momentum among particles through collisions, ionized interstellar baryonic gases. An extra pressure for the same energy density could be possible for the star gases, which have no equation of state but would introduce modifications on the equation of state of the interstellar gases. Instead, the extra term in (29) has the form of a tidal force produced by the purely geometric effect of the extra dimensions. Likewise, the equation of motion does not necessarily dismiss the influence of the extra field, as it can be illustrated by the following example. A general metric can be written for the simplified case of six dimensions as $\mathrm{d} s^{2}=-(1-2 \phi) \mathrm{d} t^{2}+\mathrm{d} \vec{x} \cdot \mathrm{d} \vec{x}+e^{-\psi} \mathrm{d} z_{1}^{2}+e^{\psi} \mathrm{d} z_{2}^{2}$ [19], where $\mathrm{d} \vec{x} \cdot \mathrm{d} \vec{x}$ is the 3 -dimensional line element. Now, in the Newtonian limit the motion is conceived to be much slower than the speed of light, $\dot{x}^{\alpha}$ can be assumed to be $(1,0,0,0)$ in (23), and the affine parameter $s$ may be approximated by the coordinate time $t$. Thus it follows that

$$
\begin{aligned}
\frac{\mathrm{d}^{2} x^{\mu}}{\mathrm{d} t^{2}}+\left\{\begin{array}{c}
\mu \\
00
\end{array}\right\} & \\
= & \frac{1}{2}\left\{\left[\frac{\partial\left(e^{-\psi}\right)}{\partial x^{\mu}}\right] e^{2 \psi} N_{z_{1}}^{2}+\left[\frac{\partial\left(e^{\psi}\right)}{\partial x^{\mu}}\right] e^{-2 \psi} N_{z_{2}}^{2}\right\} .
\end{aligned}
$$

As time derivatives of $\phi$ and $\psi$ are neglected, (31) leads to

$$
\begin{aligned}
\vec{a} & =-\nabla \Phi, \\
\Phi & =\phi+\frac{1}{2}\left(e^{-\psi} N_{z_{2}}^{2}+e^{\psi} N_{z_{1}}^{2}\right),
\end{aligned}
$$




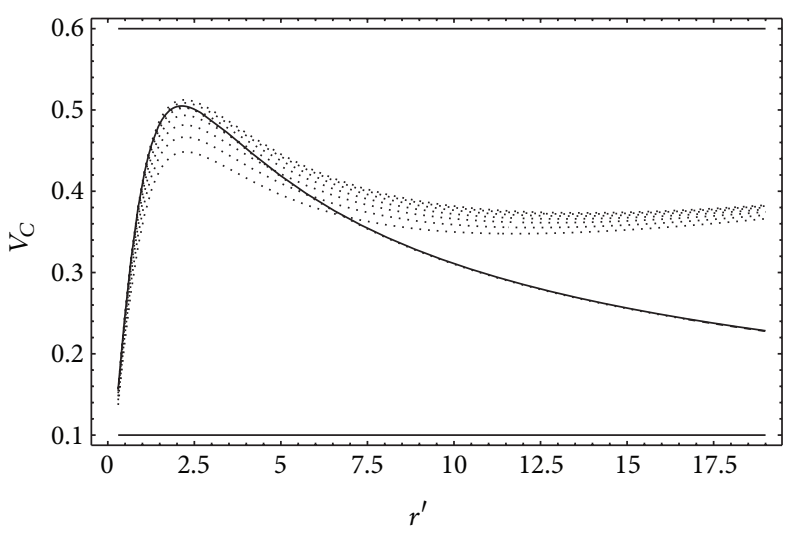

(a)

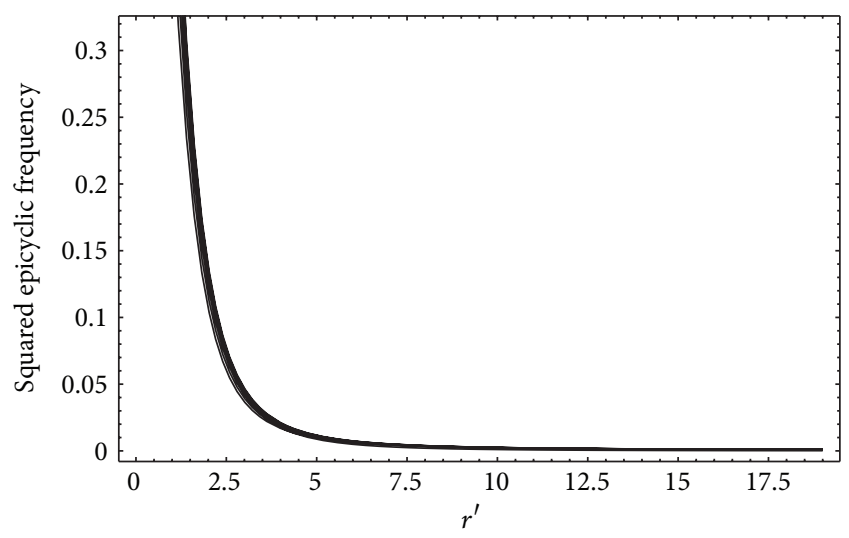

(b)

FIGURE 1: (a) The rotation curves of an effective potential where the Poisson equation is solved from a Miyamoto-Nagai ansatz, with parameters $a=1$ and $b=0.1$, describing a disk galaxy and where the Laplace equation for the extra field is solved by a Chazy-Curzon disk, with stable cut parameter $c=1.5$. They correspond to gravitating particles which orbit in a background. The full line is the curve when there are no extra dimensions; that is, $N_{z_{1}}=N_{z_{2}}=0$. The dotted lines represent a set of extra dimensional solutions for fixed $N_{z_{1}}=0.1$ and $N_{z_{2}}$ varying from 0.8 to 0.95 , as in [19]. The parameters $a, b$, and $c$ are chosen in order to make the orbital motion stable. (b) The same set of solutions presented in (a), but now we represent the squared epicyclic frequency $\kappa^{2}$. When this last is positive, we guarantee the stability of the system.

that is, the equation of motion of test bodies gravitating in an orbit in a given background for our system with an effective gravitational potential $\Phi$. To find a form for those potentials, from (28) and (30) it yields

$$
\begin{gathered}
\nabla^{2} \psi-\nabla \psi \cdot \nabla \psi=0, \\
\nabla^{2} \phi=\rho .
\end{gathered}
$$

Nonlinear terms do not appear since the $\sigma$ matrix is diagonal. In particular, (34) can be rewritten as

$$
\nabla^{2} \chi=0
$$

where the identification $\chi=e^{-\psi}$ is accomplished and hence (33) has the form

$$
\Phi=\phi+\frac{1}{2}\left(\chi N_{z_{2}}^{2}+\chi^{-1} N_{z_{1}}^{2}\right) .
$$

Together with (35) and (36), it represents a complete system of equations for a gravitational effective potential in 4dimensions, assuming a 6-dimensional universe. Note as well that (36) is the Laplace equation in flat 3-dimensional space, and therefore $\chi$ can be taken as a solution of Laplace equation for an appropriate Newtonian source of any symmetry. The form of $\phi$ can be indeed expressed as the point source potential $\sim 1 / r$ for a Newtonian system.

In a system whose acceleration $\vec{a},(32)$, has radial direction, for example, an idealized system similar to a galaxy, the rotation curves for a potential $\phi \sim-1 / r$ are provided by

$$
V_{C} \sim\left[\frac{1}{r}+\frac{1}{2} r \psi_{, r}\left(N_{z_{2}}^{2} e^{-\psi}-N_{z_{1}}^{2} e^{\psi}\right)\right]^{1 / 2} .
$$

\section{Solutions}

Now we look for a way to implement an exact Newtonian solution for the Poisson equation (35), as well as for the
Laplace equation (36). As a system similar to a galaxy is regarded, the Poisson equation can be implemented, for example, by a Miyamoto-Nagai potential, and for the case of Laplace equation a Chazy-Curzon solution can be used for an axisymmetric configuration. Namely, the MiyamotoNagai ansatz is written as [42]

$$
\phi(R, z)=-\frac{M}{\sqrt{R^{2}+\left(a+\sqrt{z^{2}+b^{2}}\right)^{2}}},
$$

where $a, b$ are positive constants. The Chazy-Curzon solution for a particle of mass $m$ in the position $z=z_{0}$ is given by $[43,44]$

$$
\chi=\frac{2 m}{R}
$$

where $R=\sqrt{r^{2}+\left(z-z_{0}\right)^{2}}$. In this last, it is possible to fix $z_{0}=$ 0 and to introduce the cut method to generate a disk solution [45], such that $z \mapsto|z|+c$, where $c$ is the cut parameter. Fixing values for $a, b$, and $c$ in such a way that $b / a \sim 0.1$, providing a similar light distribution of a disk galaxy [45], and $c>1$ (by stability issues and to prevent relativistic disks [19]) it is possible to find the set of curves given in Figure 1(a).

The stability of this last configuration is guaranteed by the positive epicyclic Newtonian parameter $\kappa^{2}=\partial^{2} \Phi / \partial r^{2}+$ $3 V_{C}^{2} / r^{2}$, as shown in Figure 1(b).

A more useful manner to analyze the effective potential is writing it as

$$
\Phi=\phi+C \cosh (\psi+\delta)
$$

where $C$ and $\delta$ are constants to be determined. This is the correspondent Newtonian potential in the present formalism. 


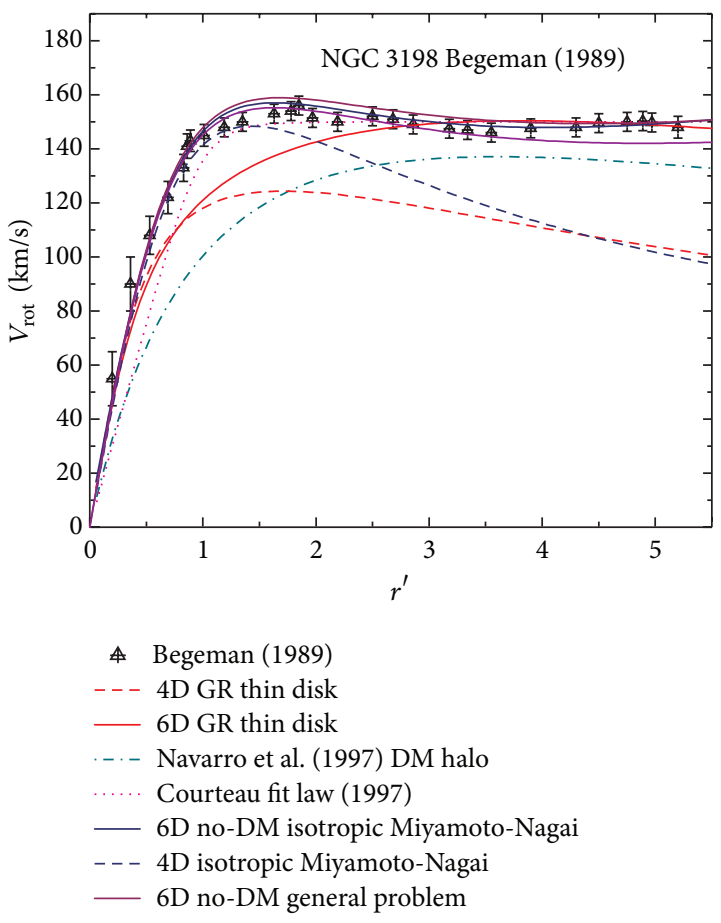

(a)

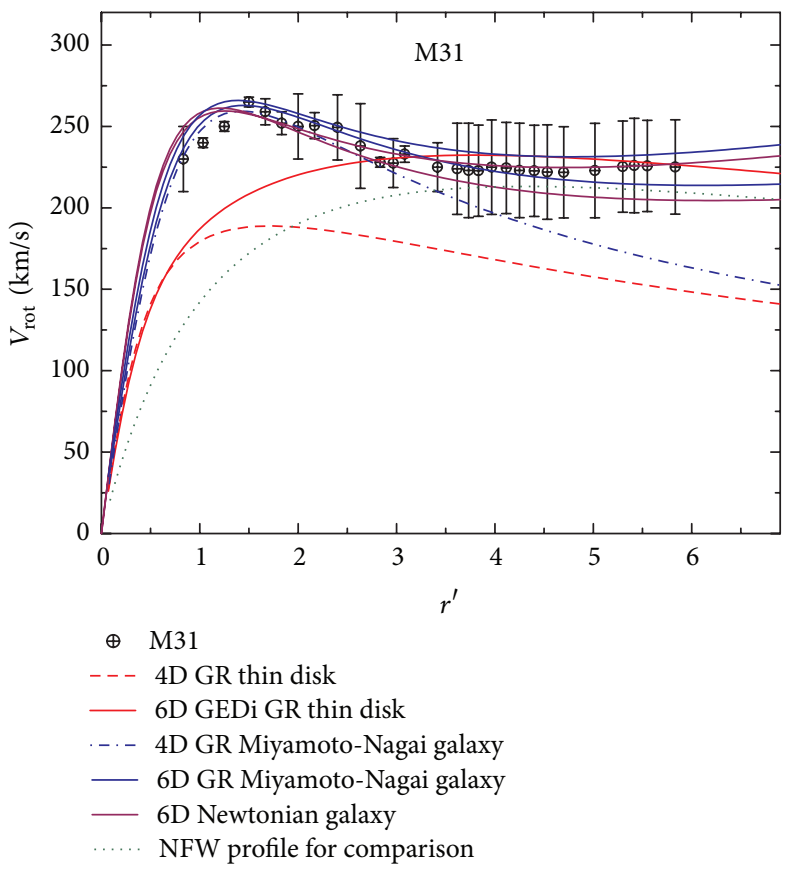

(b)

Figure 2: Values for the general 6-dimensional model (Newtonian limit): $N_{z_{1}}=0.2, N_{z_{2}}=0.8$ and $0.9, a=1$, and $b=0.01$. The violet lines are two stable examples of the present model. The full blue line is the relativistic model presented in [20].

Clearly, from (33) those constants are related to $N_{z_{1}}$ and $N_{z_{2}}$ as

$$
C^{2}=\frac{N_{z_{2}}^{2}-N_{z_{1}}^{2}}{4}, \quad \tanh \delta=\frac{N_{z_{1}}^{2}}{N_{z_{2}}^{2}} .
$$

Given the present results, one can ask about the nature of the potential (41). The term comes exclusively from extra dimensions and it may be related to a "dark matter" potential as

$$
\phi_{\text {extra } D}=\phi_{D M}=C \cosh (\psi+\delta),
$$

showing that our approach is equivalent to a dark matter effect, although the concept above is completely apart from the concept of extra matter.

\section{Probing Results with Real Galaxies}

Taking into account only the stable curves, we compare in Figures 2 and 3 some optically observed rotation curves of spiral galaxies.

Here we are not providing a composition of halo dark matter velocities plus the disk gas and the velocities of stars. What is happening is that the clean stable calculated curves are simply fitting the region of interest. The unsurprising ad hoc adjustment of $N_{z_{1}}$ and $N_{z_{2}}$ actually could assert nothing about the astrophysical role of the extra dimensions in the model. However, the calculation of stable configurations brings realizable values for $N_{z_{1}}$ and $N_{z_{2}}$, which makes it possible to visualize a minimum representation of a real disk galaxy. We furthermore compared our results to some phenomenological models used in astrophysics, as for instance in Navarro, Frenk \& White [46]. Our model is successful when compared to the observational data [47-51].

Note that our results reproduce with great fidelity the shape of nonplanar curves, as that appearing in Figure 3(b) (data taken from $[52,53]$ ).

\section{Concluding Remarks}

At the present work we showed how to calculate a "dark" potential from extradimensional imprints. If extra dimensions have some impact on the theory, certainly it is not as extra matter, but it appears at least as tidal force produced by the purely geometric effect of the extra dimensions, namely, the $(1 / 2) g^{m n} g_{m n, \alpha \beta}$ term in (29). This is confirmed by the extra force appearing in the equations of motion.

The Newtonian limit is calculated and we accomplished the visible potential that has two main components: a term from 4-dimensional metric and another term coming exclusively from extradimensional effects. The 4-dimensional term obeys the Poisson equation, and the extra term is solved from a Laplace equation. Two situations are provided: a first approximated model and a second that arises from exact solutions of Laplace and Poisson equations, respectively, the Chazy-Curzon disk solution and Miyamoto-Nagai ansatz. Those examples are calculated for a case where two extra 


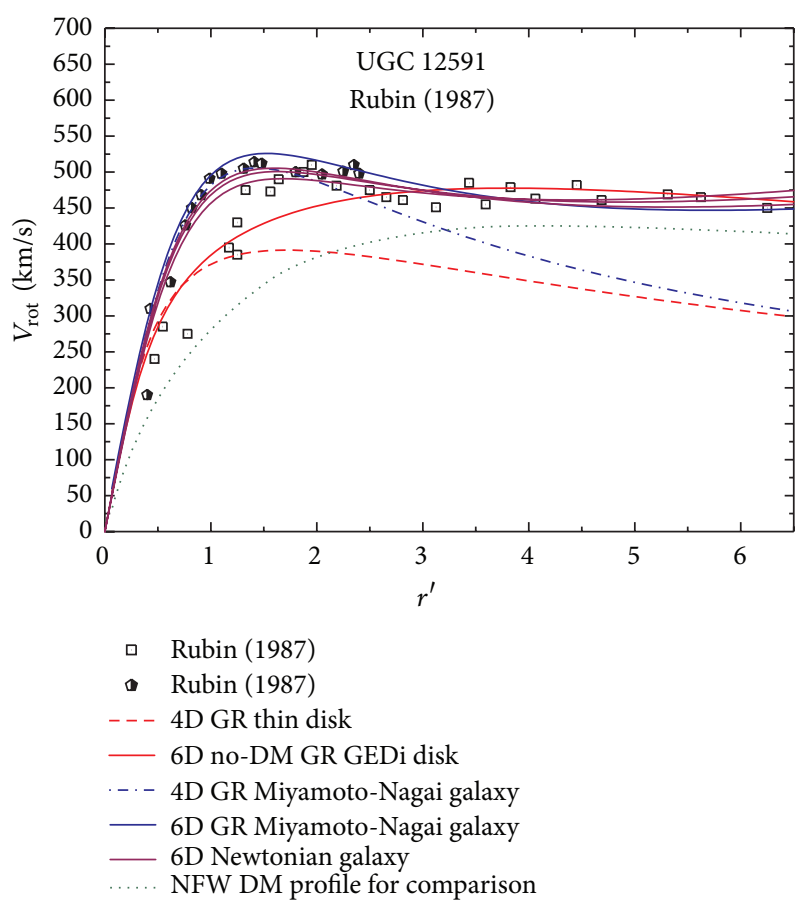

(a)

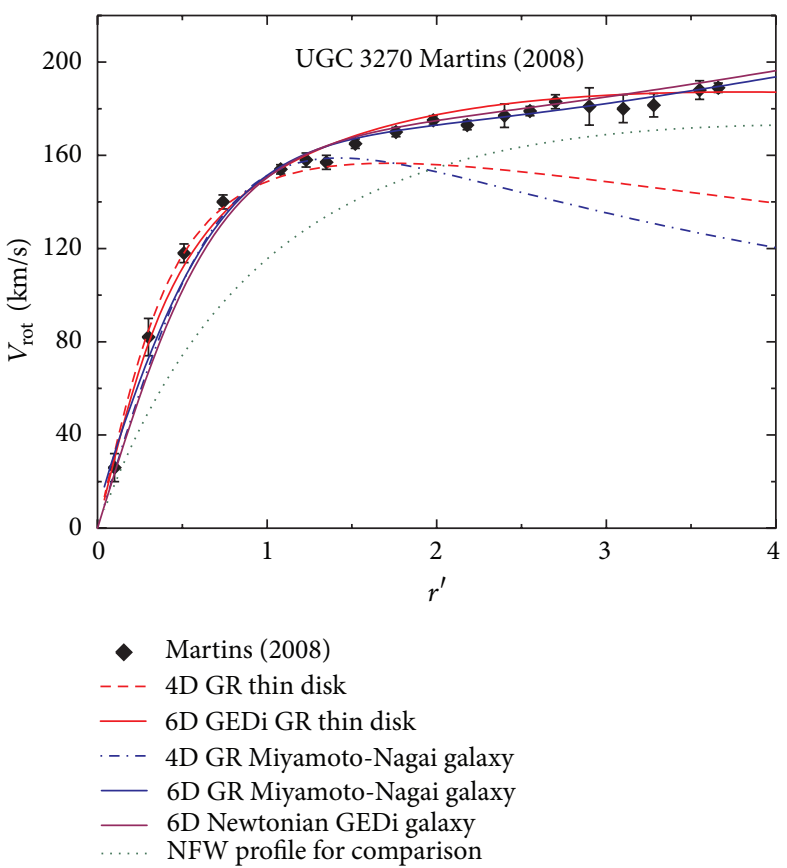

(b)

Figure 3: Values for the general 6-dimensional model: $N_{z_{1}}=0.18, N_{z_{2}}=0.85$ and $0.9, a=1$, and $b=0.01$. The violet lines are two stable examples of the present model. The full blue line is the relativistic model presented in [20].

dimensions are taken into account. Moreover, any decomposition for the weak field potential hold for this formalism, with the appropriate symmetry. For instance, as the effective models for elliptic galaxies or globular clusters, in which $\phi$ is may be a different potential.

The extra field arises naturally from metric extra terms, and the modification of gravity shown from the calculation of rotation curves can present an explanation about the missing mass effect in astronomy. The effective potential calculated here is a source to explain the phenomenological results found in $[19,20]$. The extra part of this effective potential has the form $C \cosh \left[\psi\left(x^{\alpha}\right)+\delta\right]$. The gradient of such potential represents the extra force associated with the presence of extra dimensions. It modifies the rotation curve profiles and rise anomalies in potential associated to clusters. The Laplacian corresponds to a "dark" density profile that is negligible compared to the visible density $\nabla^{2} \phi=\rho$ (see Section 6). Furthermore, the extradimensional proposal is consistent with a more general length scale and galactic morphology.

The present calculation is an alternative approach to understand the dark matter problem. In fact, to solve the dark matter problem means to solve a long list of cosmological anomalies. In this aspect, as a complement, considerations about cluster lensing are also addressed in [21-23].

\section{Acknowledgments}

The authors are extremely grateful to Patricio S. Letelier (in memoriam) for the important and profound contribution concerning interpretation and discussion of the present results. The work of C. H. Coimbra-Araújo is supported by Fundação Araucária and PDEE/CAPES Programme under Grant no. 3874-07-9. R. da Rocha is grateful to $\mathrm{CNPq}$ 472903/2008-0 and 304862/2009-6 for financial support.

\section{References}

[1] L. Randall and R. Sundrum, "Large mass Hierarchy from a small extra dimension," Physical Review Letters, vol. 83, no. 17, pp. 3370-3373, 1999.

[2] L. Randall and R. Sundrum, "An alternative to compactification," Physical Review Letters, vol. 83, no. 23, pp. 4690-4693, 1999.

[3] H. Leutwyler, "Solution statique à symmetrie spherique en theorie penta-dimensionnelle," Archives des Sciences, vol. 13, article 549, 1960.

[4] P. Dobiasch and D. Maison, "Stationary, spherically symmetric solutions of Jordan's unified theory of gravity and electromagnetism," General Relativity and Gravitation, vol. 14, no. 3, pp. 231-242, 1982.

[5] D. Pollard, "Antigravity and classical solutions of fivedimensional Kaluza-Klein theory," Journal of Physics A, vol. 16, no. 3, pp. 565-574, 1983.

[6] A. Chodos and S. Detweiler, "Spherically symmetric solutions in five-dimensional general relativity," General Relativity and Gravitation, vol. 14, no. 10, pp. 879-890, 1982.

[7] G. W. Gibbons and D. L. Wiltshire, "Black holes in KaluzaKlein theory," Annals of Physics, vol. 167, no. 1, pp. 201-223, 1986, Erratum in: Annals of Physics, 176, 393, 1986. 
[8] G. W. Gibbons and M. J. Perry, "Soliton supermultiplets and Kaluza-Klein theory," Nuclear Physics B, vol. 248, no. 3, pp. 629646, 1984.

[9] M. Cvetic and D. Youm, "Static four-dimensional Abelian black holes in Kaluza-Klein theory," Physical Review D, vol. 52, pp. 2144-2149, 1995.

[10] M. Cvetič and D. Youm, "All the four-dimensional static, spherically symmetric solutions of Abelian Kaluza-Klein theory," Physical Review Letters, vol. 75, no. 23, pp. 4165-4168, 1995.

[11] D. Rasheed, “The rotating dyonic black holes of Kaluza-Klein theory," Nuclear Physics B, vol. 454, no. 1-2, pp. 379-401, 1995.

[12] J. M. Overduin and P. S. Wesson, "Kaluza-Klein gravity," Physics Reports, vol. 283, no. 5-6, pp. 303-378, 1997.

[13] P. Brax and C. van de Bruck, "Cosmology and brane worlds: a review," Classical and Quantum Gravity, vol. 20, no. 9, article R201, 2003.

[14] R. Maartens, “Brane-world gravity," Living Reviews in Relativity, vol. 7, article 7, 2004.

[15] T. Gherghetta and M. Shaposhnikov, "Localizing gravity on a string-like defect in six dimensions," Physical Review Letters, vol. 85 , no. 240, p. 243, 2000.

[16] A. G. Cohen and D. B. Kaplan, "Solving the hierarchy problem with noncompact extra dimensions," Physics Letters B, vol. 470, pp. 52-58, 1999.

[17] R. Gregory, "Nonsingular global string compactifications," Physical Review Letters, vol. 84, pp. 2564-2567, 2000.

[18] V. A. Rubakov, "Large and infinite extra dimensions," PhysicsUspekhi, vol. 44, no. 9, pp. 871-893, 2001.

[19] C. H. Coimbra-Araújo and P. S. Letelier, "On the stability of universal extradimensional disks," Classical and Quantum Gravity, vol. 25, no. 1, Article ID 015001, 2008.

[20] P. S. Letelier and C. H. Coimbra-Araujo, "Gravity with extra dimensions and dark matter interpretation: phenomenological example via Miyamoto-Nagai galaxy," Brazilian Journal of Physics, vol. 42, no. 1-2, pp. 100-109, 2012.

[21] C. H. Coimbra-Araujo and P. S. Letelier, "Athin disk in higher dimensional space-time and dark matter interpretation," Physical Review D, vol. 76, no. 4, Article ID 043522, 17 pages, 2007.

[22] C. H. Coimbra-Araujo and P. S. Letelier, "General relativistic results for a galactic disc in a multidimensional space-time," in Proceedings of the IAU Symposium n. 238, V. Karas, Ed., Cambridge University Press, Cambridge, UK, 2006.

[23] C. H. Coimbra-Araujo and P. S. Letelier, "A general relativistic model of galaxy bulges in GEDi paradigm," in Proceedings of the IAU Symposium n. 245, M. Bureau, E. Athanassoula, and B. Barbuy, Eds., pp. 239-240, Cambridge University Press, Cambridge, UK, 2007.

[24] M. E. Kahil and T. Harko, "Is dark matter an extra-dimensional effect?” Modern Physics Letters A, vol. 24, no. 9, pp. 667-682, 2009.

[25] T. Appelquist, H. C. Cheng, and B. A. Dobrescu, "Bounds on universal extra dimensions," Physical Review D, vol. 64, no. 3, Article ID 035002, 10 pages, 2001.

[26] D. Hooper and S. Profumo, "Dark matter and collider phenomenology of universal extra dimensions," Physics Reports, vol. 453, no. 2-4, pp. 29-115, 2007.

[27] R. Emparan, G. T. Horowitz, and R. C. Myers, "Black holes radiate mainly on the brane," Physical Review Letters, vol. 85, pp. 499-502, 2000.
[28] V. Cardoso, M. Cavaglia, and L. Gualtieri, "Black hole particle emission in higher-dimensional spacetimes," Physical Review Letters, vol. 96, no. 7, Article ID 071301, 4 pages, 2006, Erratum in: Physical Review Letters, 96, 219902, 2006.

[29] V. Cardoso, M. Cavaglia, and L. Gualtieri, "Hawking emission of gravitons in higher dimensions: non-rotating black holes," Journal of High Energy Physics, vol. 2006, no. 2, article 021, 2006.

[30] R. C. Myers and M. J. Perry, "Black holes in higher dimensional space-times," Annals of Physics, vol. 172, no. 2, pp. 304-347, 1986.

[31] V. Frolov and D. Stojkovic, "Black hole as a point radiator and recoil effect on the brane world," Physical Review Letters, vol. 89, no. 15, Article ID 151302, 2002.

[32] R. Emparan, J. Garcia-Bellido, and N. Kaloper, "Black hole astrophysics in AdS braneworlds," Journal of High Energy Physics, vol. 2003, no. 1, article 079, 2003.

[33] A. N. Aliev and A. E. Gumrukcuoglu, "Gravitational field equations on and off a 3-brane world," Classical and Quantum Gravity, vol. 21, no. 22, pp. 5081-5096, 2004.

[34] G. Duffy, C. M. Harris, P. Kanti, and E. Winstanley, "Brane decay of a $(4+n)$-dimensional rotating black hole: spin-0 particles," Journal of High Energy Physics, vol. 2005, no. 9, article 049, 2005.

[35] R. da Rocha and C. H. Coimbra-Araujo, "Extra dimensions at the CERN LHC via mini-black holes: effective Kerr-Newman brane-world effects," Physical Review D, vol. 74, no. 5, Article ID 055006, 12 pages, 2006.

[36] R. da Rocha and C. H. Coimbra-Araujo, "Variation in the luminosity of Kerr quasars due to an extra dimension in the brane Randall Sundrum model," Journal of Cosmology and Astroparticle Physics, vol. 2005, no. 12, article 009, 2005.

[37] R. Gregory, V. A. Rubakov, and S. M. Sibiryakov, "Brane worlds: the gravity of escaping matter," Classical and Quantum Gravity, vol. 17, pp. 4437-4449, 2000.

[38] J. M. H. da Silva and R. da Rocha, "Torsion effects in braneworld scenarios," Physical Review D, vol. 81, no. 2, Article ID 024021, 8 pages, 2010.

[39] M. C. B. Abdalla, J. M. Hoff da Silva, and R. da Rocha, "Notes on the two-brane model with variable tension," Physical Review $D$, vol. 80, Article ID 046003, 2009.

[40] K. A. Bronnikov, V. N. Melnikov, and H. Dehnen, "General class of brane-world black holes," Physical Review D, vol. 68, Article ID 024025, 2003.

[41] R. M. Wald, General Relativity, The University of Chicago Press, Chicago, Ill, USA, 1984.

[42] M. Miyamoto and N. Nagai, "Three-dimensional models for the distribution of mass in galaxies," Astronomical Society of Japan, vol. 27 , no. 4 , pp. 533-543, 1975.

[43] M. Chazy, "Sur le champ de gravitation de deux masses fixes dans la théorie de la relativité," Bulletin de la Société Mathématique de France, vol. 52, pp. 17-38, 1924.

[44] H. Curzon, "Cylindrical solutions of Einstein's gravitation equations," Proceedings of the London Mathematical Society, vol. 23, pp. 477-480, 1925.

[45] J. Binney and S. Tremaine, Galactic Dynamics, Princeton University Press, Princeton, NJ, USA, 1987.

[46] J. F. Navarro, C. S. Frenk, and S. D. M. White, "A universal density profile from hierarchical clustering," Astrophysical Journal Letters, vol. 490, no. 2, pp. 493-508, 1997.

[47] Y. Sofue, Y. Tutui, M. Honma et al., "Central rotation curves of spiral galaxies," Astrophysical Journal Letters, vol. 523, no. 1, pp. 136-146, 1999. 
[48] V. Rubin, "Constraints on the dark matter from optical rotation curves," in Proceedings of the IAU Symposium n. 117, pp. 51-62, D. Reidel, Dordrecht, The Netherlands, 1987.

[49] K. G. Begeman, "H I rotation curves of spiral galaxies. I-NGC, 3198," Astronomy and Astrophysics, vol. 223, no. 1-2, pp. 47-60, 1989.

[50] O. Garrido, M. Marcelin, and P. Amram, "GHASP: an Ha kinematic survey of spiral and irregular galaxies-III. 15 new velocity fields and study of 46 rotation curves," Monthly Notices of the Royal Astronomical Society, vol. 349, no. 1, pp. 225-239, 2004.

[51] Y. Sofue, J. Koda, H. Nakanishi, and S. Onodera, "The virgo high-resolution CO survey: II. Rotation curves and dynamical mass distributions," Publications of the Astronomical Society of Japan, vol. 55, p. 59, 2003.

[52] C. F. Martins and P. Salucci, "Analysis of rotation curves in the framework of the gravitational suppression model," Physical Review Letters, vol. 98, no. 15, Article ID 151301, 2007.

[53] C. F. Martins and P. Salucci, "Analysis of rotation curves in the framework of $R^{n}$ gravity," Monthly Notices of the Royal Astronomical Society, vol. 381, pp. 1103-1108, 2007. 

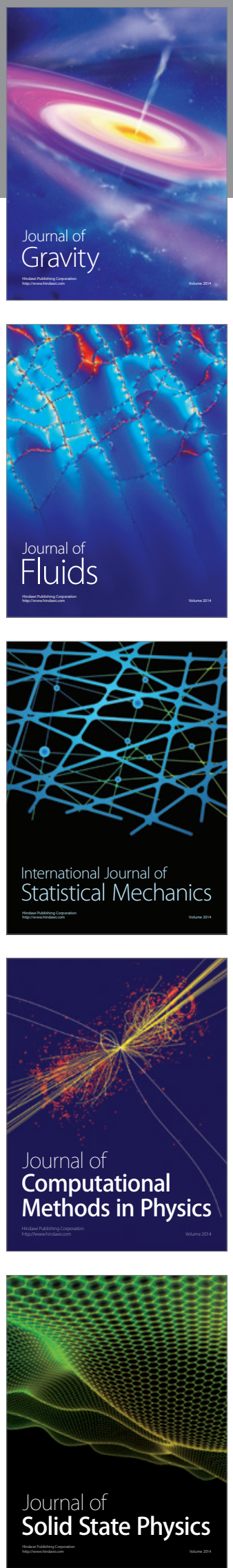

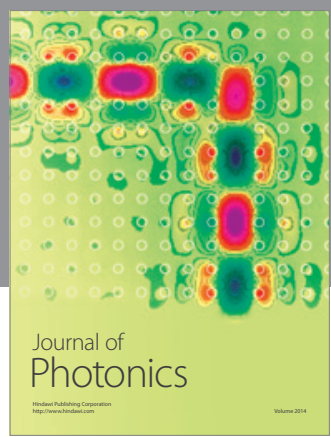

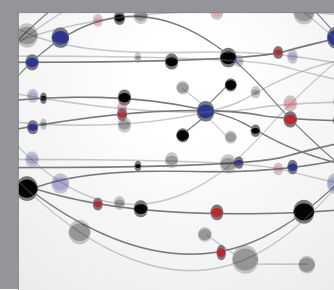

The Scientific World Journal

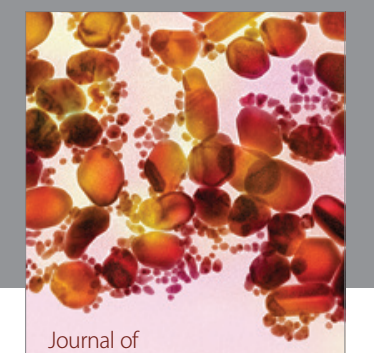

Soft Matter
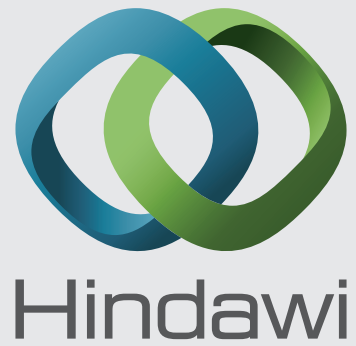

Submit your manuscripts at

http://www.hindawi.com
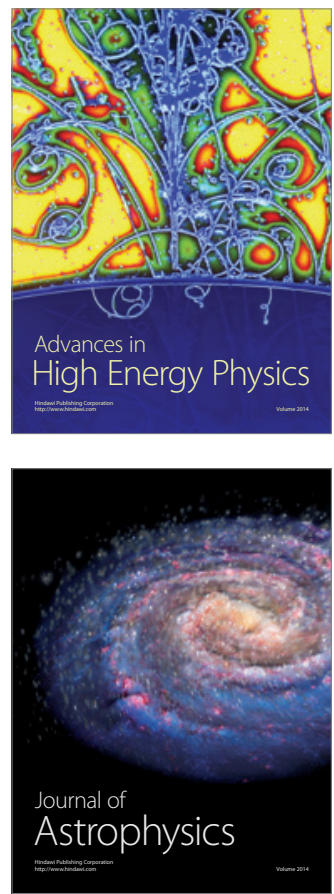
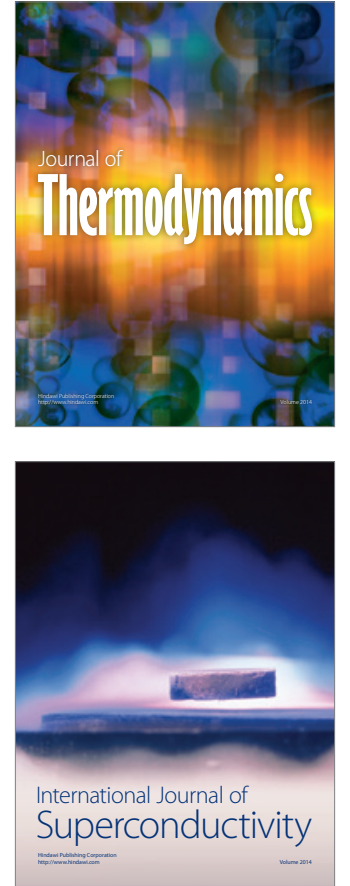
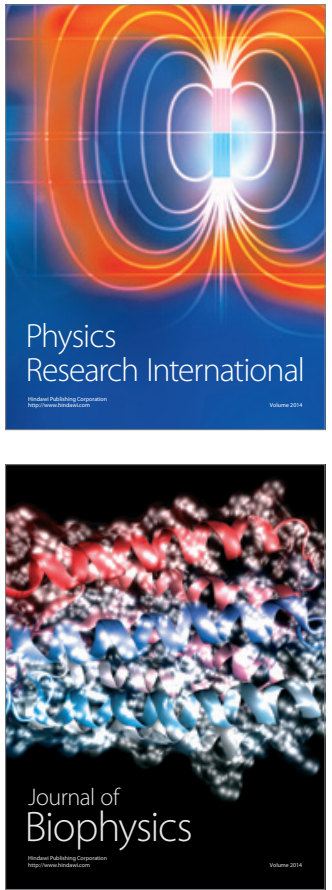
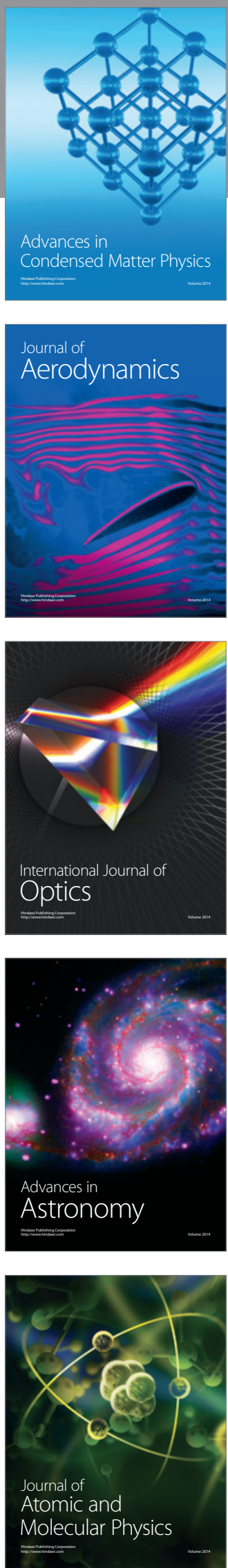